\title{
Информачионная поддержка этапа разработки состава твердых лекарственных форм
}

\author{
B.Ф. Корнюшко 1, д.m.н., nрофрессор, vfk256@mail.ru \\ O.M. Николаева 1, ассистент, polyakova@mitht.ru \\ И.В. Богунова 1, научный сотрудник, irina_bogunova@mail.ru \\ A.A. Флид 1, ведущий программист, a.a.flid@gmail.com \\ А.А. Гребеншиков 1, магистр, me@package.su
}

1 Российский технологический университет МИРЭА (Институт тонких химических технологий им. М.В. Аомоносова), г. Москва, 119454, Россия

В статье рассматривается применение системного анализа для информационной поддержки этапа разработки состава твердых лекарственных форм. Приведена диаграмма информационного описания жизненного цикла разработки и производства лекарственных препаратов в номинации IDEF0. В основу построения системы информационной поддержки этапа разработки состава в жизненном цикле положен новый подход QbD (quality by design, «качество, запланированное при разработке»), который должен быть реализован на основе целевого профиля препарата TPP (target product profile).

В качестве критериев оценки качества оптимизации на всех стадиях разработки выбраны технологические параметры, характеризующие основные свойства полученных промежуточных продуктов, СРP (critical process parameter).

Рассмотрена схема интеллектуальной системы управления этапом разработки состава готовой лекарственной формы.

Для управления исследованиями предлагаются два алгоритма: один используется при подборе вспомогательных веществ, а второй при выборе оптимальных технологических параметров для каждой стадии.

Подробно рассмотрено построение на основе многооткликовой регрессионной модели алгоритма подбора параметров всех процессов, участвующих в получении оптимального состава готовой лекарственной формы.

Рассмотрено построение БД вспомогательных веществ, входящей в структуру интеллектуальной системы управления. Приводятся схемы, демонстрирующие основные наборы сущностей и связи между ними. Помимо прочего, приведены некоторые фрагменты программного кода, подкрепляющие теоретическое обоснование проводимого исследования.

Ключевые слова: системный анализ, разработка состава, фармачевтическая разработка, информачионная поддержка, номиначия IDEF0, подход QbD, профиль TPP, критерий, информационная модель, БД, система управления.

Одним из важнейших направлений применения компьютерных технологий в современном информационном оснащении крупных производственных, образовательных и научных объединений, позволяющих интегрировать их информационные ресурсы, является создание единых информационных систем (ЕИС), которые составят основу единой информационной среды соответствующего объединения, отрасли и т.д. (университетские информационные сети, сети организаций здравоохранения и другие), в частности, системы непрерывного информационного обеспечения информационной среды в сфере производства лекарственных препаратов (ЛП).

Разработка каждого нового ЛП обычно требует больших финансовых затрат, зачастую превышающих 100 млн долларов, и значительного времени 8-10 лет. Жизненный цикл производства готового ЛП включает много этапов, в которых принимают участие сотни исследователей: медики, биохимики, химики, технологи, математики, компьютерщики. И тысячи людей привлекаются на этапах доклинических и клинических испытаний разработанной готовой лекарственной формы (ГЛФ). Исторически построению информационных систем на этапе клинических исследований всегда уделялось особое внимание и отдавалось предпочтение, а следовательно, именно на него направлялись материальные и финансовые ресурсы [1].

Более того, по данным иностранных литературных источников, для абсолютного большинства этапов жизненного цикла разработки и производства ЛП разработаны и внедрены системы управления качеством продукции для доклинических исследований - GLP, клинических исследований GCP, производства ЛП - GMP, хранения ЛП - GSP, дистрибьюции - GDP, отпуска в аптеки - GPP. Таким образом, практически все этапы жизненного цикла ЛП оснащены системами управления качеством, кроме этапа фармацевтической разработки, методическому и информационному обеспечению которого, на взгляд авторов, уделялось гораздо меньше внимания. Однако в последнее время появился совершенно новый подход, позволяющий надеяться на то, что эта проблема может быть разрешима. Сущность нового подхода состоит в применении на стадии разработки ЛП принципа QbD (quality by design, «качество, запланированное при разработке») [2, 3], что в будущем позволит сформулировать основы построения систем управления 
качеством для этапа разработки состава ГЛФ и на основе системного подхода решать огромную проблему построения ЕИС на всех этапах жизненного цикла ЛП.

На рисунке 1 приведена информационная модель разработки состава твердой лекарственной формы (ТЛФ) ЛП от молекулярного моделирования до опытно-промышленного производства. Информационная модель выполнена в нотации IDEF0 и позволяет установить информационные связи этапов разработки.

Как отмечено в [2], разрабатываемая форма должна быть не только эффективной и безопасной, но и технологичной, а также физически и химически стабильной от начала производства до конца срока хранения. Решению этих задач, по мнению авторов монографии, способствует принятие подхода $\mathrm{QbD}$, который должен обеспечивать создание препарата заданного качества с минимальным риском. Основными в нем являются экспериментальные исследования, в которых, в свою очередь, важнейшее значение имеет аналитическое и информационное сопровождение исследований, необходимое для контроля самого технологического процесса и выпускаемого препарата.

В монографии [2] приведены исследования десятков авторов, посвященных биохимическим, химическим, технологическим и математическим проблемам производства ЛП, однако абсолютно не затронут очень важный информационный аспект. Этот аспект сравнительно мало освещается в литературе, хотя можно выделить работу [4], в которой подробно исследуются проблемы построения базы вспомогательных веществ (ВВ), выбора оборудования и трансфера технологических операций. Авторы данной статьи пытаются, используя системный подход, хотя бы в малой мере осветить проблемы построения единой информационной поддержки фармацевтической разработки ГЛФ одного из видов ЛП в твердой форме.

Для этого в работе рассмотрено решение следующих основных задач:

- обоснование выбора глобального и локальных критериев оптимизации проводимых исследований;

- применение методов и средств системного моделирования, основанных на методологии структурного анализа и проектирования SADT, для построения функциональных моделей, отображающих информационные связи между элементами системы [5];

- применение математических моделей для решения задач управления и оптимизации.

Подход QbD должен быть реализован на основе целевого профиля препарата ТPP (target product profile), представляющего собой оптимальную со-

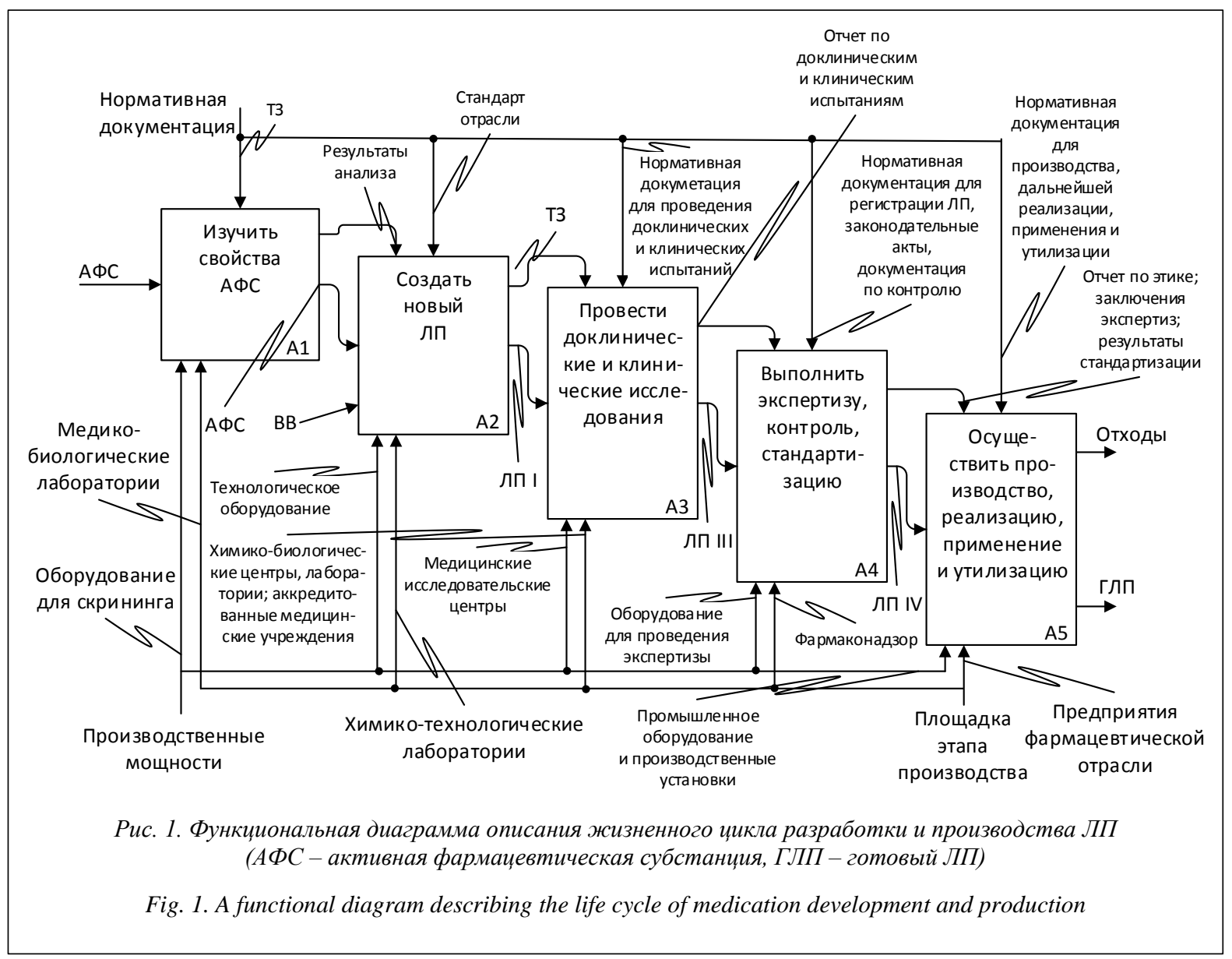


вокупность параметров, обеспечивающих препарату оптимальную эффективность, схему лечения и коммерческий потенциал [6].

Как отмечено в [7], полная стратегия разработки ЛП базируется на минимизации риска в областях безопасности, качества и бизнеса. Одним из эффективных подходов является минимизация рисков на стадии фармацевтической разработки, что позволяет в дальнейшем минимизировать их при трансфере технологии.

Фармацевтическая разработка ГЛФ проходит ряд стадий на двух уровнях, обычно проводимых в различных лабораториях разными группами исследователей [8]: выбор лидерного вещества и разработка на его основе ГЛФ.

На рисунке 2 представлена информационная модель этапа разработки ГЛФ в номинации IDEF0. Первый уровень - синтез активной фармацевтической субстанции (АФС), который проходит в непрерывных проточных реакторах, что помогает избежать больших временных затрат.
Данная технология позволяет осуществлять контроль на всех стадиях синтеза. Выбор АФС производится в соответствии с техническим заданием (Т3) из ряда претендентов, задаваемых специализированной БД. Второй уровень - подбор ВВ, выбор технологической платформы, а также дальнейшая разработка состава ГЛФ по третьему блоку [4].

На рисунке 3 приведена информационная модель фармацевтической разработки ГЛФ на основе функционального моделирования посредством IDEF0-моделей [8]. На диаграмме отражены основные функциональные модули разработки ГЛФ.

Дочерние диаграммы декомпозиции уровней позволяют подробно исследовать связи внутри каждого из технологических модулей.

Построение системной информационной модели, реализующей принцип QbD, опирается на поиск оптимального варианта (программы) проводимых исследований, который всегда подразумевает наличие критерия оптимизации.

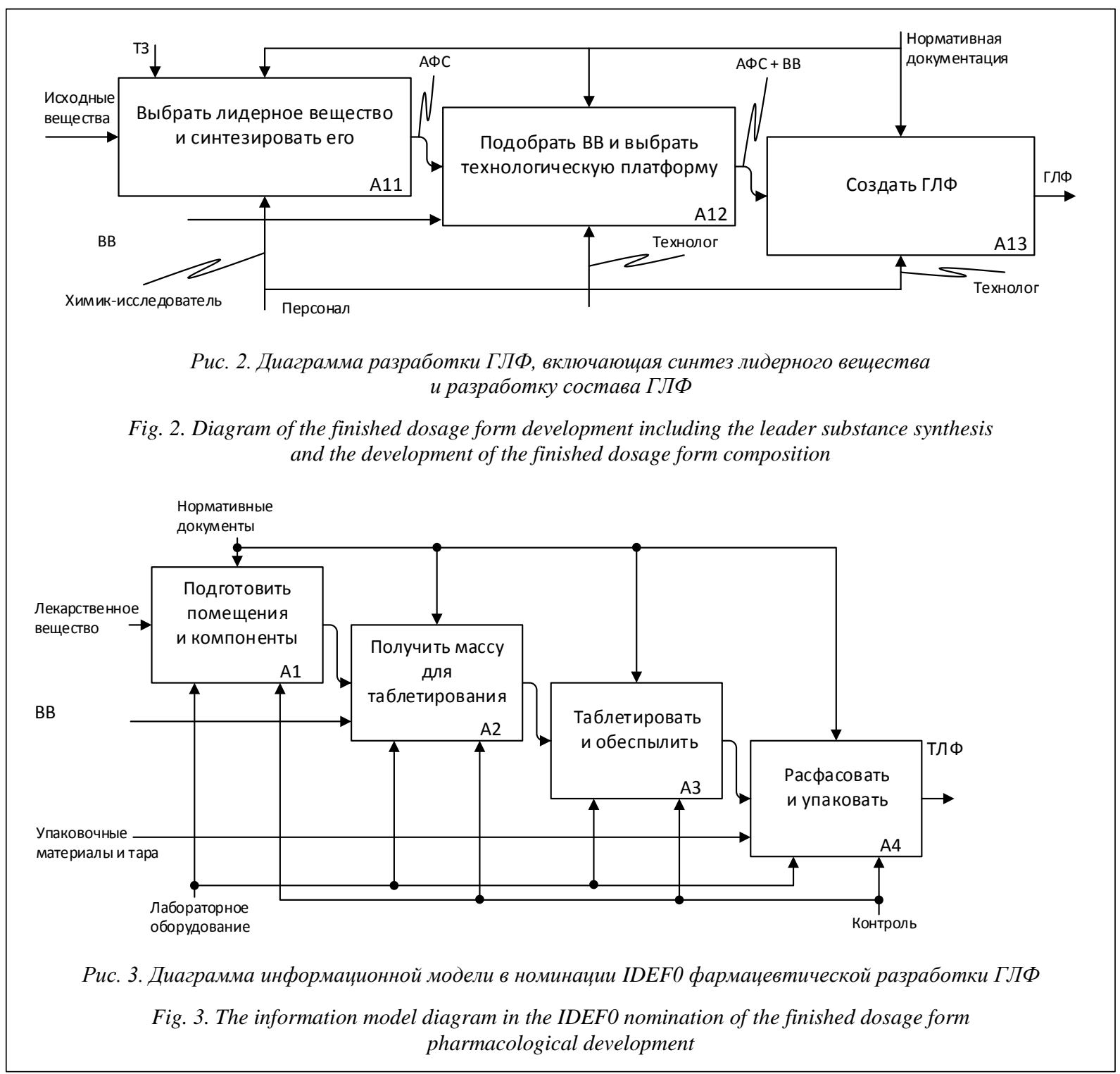


В [2] дано понятие некоторого глобального критерия CQA (critical quality attribute) - количественное определение свойства продукта, которое считается критическим для определения чистоты, эффективности и безопасности продукта. Этот критерий, в свою очередь, представляет собой некоторую взвешенную сумму локальных критериев, оценивающих эффективность отдельных технологических переделов этапа разработки. При этом каждый из критериев $k_{i}$, называемых локальными, характеризует некоторую локальную цель общей технологической задачи, достигаемую при выполнении определенных условий. Обозначим глобальную цель проводимой разработки как интегральный критерий $K_{g}$, образованный по тем или иным правилам локальными критериями и характеризующий общее направление изменения плана исследования в зависимости от достигнутых результатов.

Процесс управления разработкой характеризуется необходимостью реализации управляющих воздействий в форме определенных мероприятий, отражаемых в маршрутных картах исследователя, $M=\left\{m_{i}\right\}$, где $i=1, \ldots, q(q-$ количество мероприятий), для выполнения которых с определенной интенсивностью $V=\left\{v_{i}\right\}$, где $i=1, \ldots, q$, используются некоторые ресурсы $R=\left\{r_{i}\right\}$. Конкретное мероприятие можно охарактеризовать кортежем $m_{i}=\left\{v_{i}, r_{i}\right\}$.

Выбор принципа оптимальности управленческого решения всегда зависит от предпочтений ЛПР, а также от особенностей поставленной задачи, является активным и компромиссным [9].

Оптимальный в представленных условиях вариант решения $\overline{K_{g}}$ должен удовлетворять соотношению

$$
\overline{K_{g}}(\bar{X})=\underset{M \in W_{X}}{\operatorname{optim}}(K(X), A),
$$

где $\overline{K_{g}}$ - оптимальное значение интегрального критерия; optim - оператор, определяющий выбранный метод оптимизации.

При выборе оптимального варианта используется принцип гибкого приоритета: оценка вариантов производится по взвешенному векторному критерию. Поэтому естественным можно считать упрощение модели за счет перехода от пространства выбираемых решений $W_{X}$ к пространству допустимых локальных критериев $W_{K}$, а затем перехода от пространства допустимых локальных критериев к пространству индикаторов $W_{I}$.

С точки зрения вклада в процесс анализа каждый переход обеспечивает выполнение своей уникальной функции. Критериальная оценка позволяет сравнить варианты решения на соответствие предъявленным требованиям относительно рассчитанной величины интегрального критерия. Индикативная оценка дает возможность определить уровень критичности состояния изучаемого объекта в случае реализации выбранного варианта. Следует отметить, что критериальная оценка является более точной по сравнению с индикативной, что определяет предпочтительные области их применения. Выбор вида оценки целесообразно осуществлять в каждом конкретном случае.

Индикативная оценка позволяет наглядно отобразить уровень критичности в соответствии с разработанной шкалой. На основе такой шкалы рассчитывается интегральный балл, включающий в себя все типы параметров. Индикативная оценка задается в виде целевой функции, указывающей полезность (ценность, эффективность) того исхода, который получается в ситуации, когда принимающий решение выбирает соответствующую альтернативу.

В качестве критериальных оценок на этапе фармацевтической разработки ЛП выбраны CQA, характеризующие на каждом технологическом переделе такое свойство полученного промежуточного продукта, которое считается критическим для заданных ограничений чистоты, безопасности и эффективности продукта. В качестве индикативных оценок используются CPP (critical process parameter) - технологические показатели, которые при выходе за рамки ограничений, задаваемых нормативными документами, оказывают прямое влияние на критериальную оценку.

Значение каждого из критериев оценивается соответствующим профессиональным экспертом, и далее на основании их оценок чаще всего расчетным путем определяется глобальный критерий многокритериальная экспертная оценка. Тогда глобальный критерий $G$ может быть определен через множество частных критериев $K_{j}^{m}$ :

$$
G=\left\{S_{i}^{m} \mid i=\overline{1, M}\right\} \stackrel{f^{P}}{\longrightarrow} K^{M}=\left\{K_{j}^{m}, D_{j}^{m} \mid j=\overline{1, N}\right\},
$$

где $S_{i}^{m}$ - элемент некоторой формальной модели; $f^{P}$ - функция отображения; $N$ - число введенных частных критериев; $D_{j}^{m}-$ множество атрибутов частного критерия (наименование, описание и т.д.).

В процессе управления исследованиями фармацевтической разработки ТЛФ все больше участвуют интеллектуальные системы управления, позволяющие производить сбор, хранение и обработку информации, а также готовить варианты управленческих решений для лиц, принимающих решения [10]. На рисунке 4 представлена схема одного из вариантов реализации интеллектуальной информационной системы управления разработкой ЛП в твердой форме.

Важнейшими задачами на этапе фармацевтической разработки являются выбор ВВ, определение технологической платформы с технологическими процессами и соответствующим оборудованием, а также определение параметров проведения технологических процессов. 


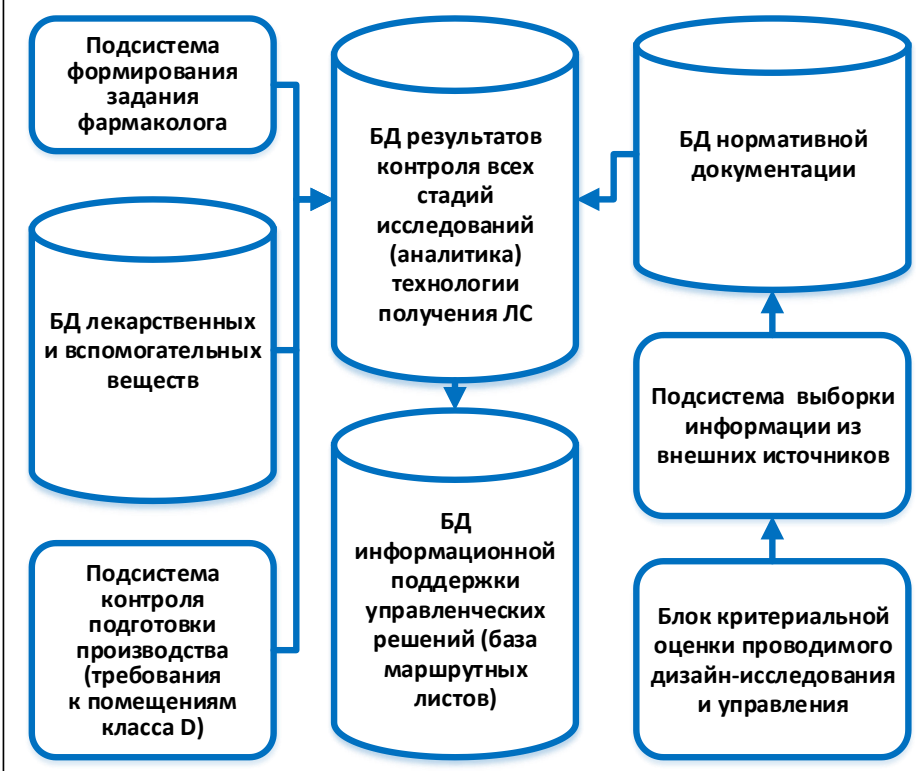

Рис. 4. Схема интеллектуальной системы управления этапом разработки состава ГЛФ

Fig. 4. The diagram of the intellectual control system of the development stage of the finished dosage form composition

Для управления исследованиями предлагаются два алгоритма: один используется при подборе ВВ, а другой - при выборе оптимальных технологических параметров для каждой стадии. Алгоритм выбора ВВ из БД ВВ представляет собой цепочку логических операторов для каждой стадии процесса (рис. 5).

Работу алгоритма можно описать с помощью оператора условного перехода:

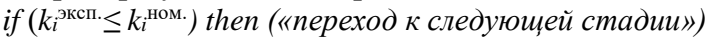
else («возврат к началу прочесса и задание нового ВВ»);

Если на стадии определяются два критерия (измеряются два коэффициента оценивания качества смеси), алгоритм несколько усложняется:

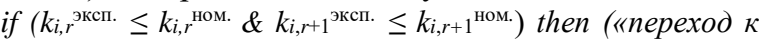
следующей стадии»)

else («возврат к началу прочесса и задание нового ВВ»)

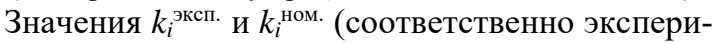
ментально найденное и номинальное, задаваемое в нормативных документах) критериального коэффициента, оценивающего качество смеси на $i$-й стадии; $i$ - номер стадии $(i=1, \ldots, I) ; r=1,2$ - номер измеряемого критериального коэффициента на $i$-й стадии, если их более одного.

Если ВВ не удается найти с первого раза, то есть

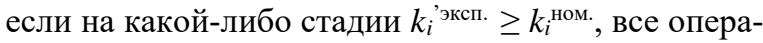
ции поиска оптимального состава смеси начинаются сначала.

Второй разработанный алгоритм управления используется для подбора параметров всех процессов, участвующих в получении ЛВ оптимального состава. При этом в соответствии с принципом $\mathrm{QbD}$ эти параметры должны оптимизироваться.
Будем подбирать параметры технологических процессов, задействованных в разработке, опираясь на методы оптимизации и регрессионные математические модели вида

$Y=\sum b_{i} x_{i}+\sum \sum b_{i j} x_{i j}(i=1,2, \ldots, n ;$ $j=1, \ldots, m ; i \neq j ; j \geq i$.

Коэффициенты $x_{i j}$ при этом представляют собой соответствующие технологические параметры для каждого передела. Например, для операции влажного гранулирования в качестве $x_{1}$ может быть использован такой параметр, как число циклических повторений, а $x_{2}-$ скорость вращения мешалки и т.д. Важнейшее значение при построении модели имеет критерий оптимизации $Y$. Как отмечалось выше, глобальным критерием оптимизации является критерий CQA некоторое количественное определение свойства продукта, которое считается критическим для определения чистоты, эффективности и безопасности продукта. Этот критерий определяется на основании информации, поступающей не только на этапе разработки, но и по данным доклинических и клинических испытаний на протяжении нескольких лет. Для оперативного управления служат локальные критерии, которые на различных стадиях фармацевтической разработки оценивают качество получаемой смеси с различных сторон. Так как напрямую это качество оценить нельзя, используют косвенные критерии, количественно определяемые через данные контроля на каждой стадии. Это дает возможность построить регрессионные модели каждой технологической стадии разработки, применив в качестве независимых управляемых переменные $x_{i j}$ - технологические параметры соответствующих процессов, а в качестве целевой функции $y$ - экспериментально найденные значения показателей кон-

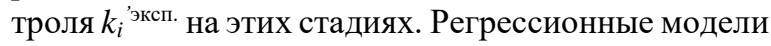
для исследуемых процессов имеют следующие особенности:

- в ряде стадий используются два метода контроля, а не один, поэтому в общем случае необходимо рассматривать многооткликовые модели регрессии:

$Y_{i}^{k}=\sum b_{i}{ }^{k} x_{i}+\sum \sum b_{i j}{ }^{k} x_{i j}(i=1,2, \ldots, n ; j=1, \ldots, m ;$ $i \neq j ; j \geq i ; k=1,2)$;

- регрессионные коэффициенты $b_{i}{ }^{k}, b_{i j}{ }^{k}$ относятся к модели с откликом $Y_{i}^{k}$.

При регрессионном вычислении коэффициентов $b_{i}{ }^{k}, b_{i j}{ }^{k}$ необходимо учесть ограничения на целевые функции: $Y_{i}^{k \min } \leq Y_{i}^{k} \leq Y_{i}^{k \max }$.

Для подбора оптимальных значений параметров $x_{i}$ используются численные методы условной оптимизации. 


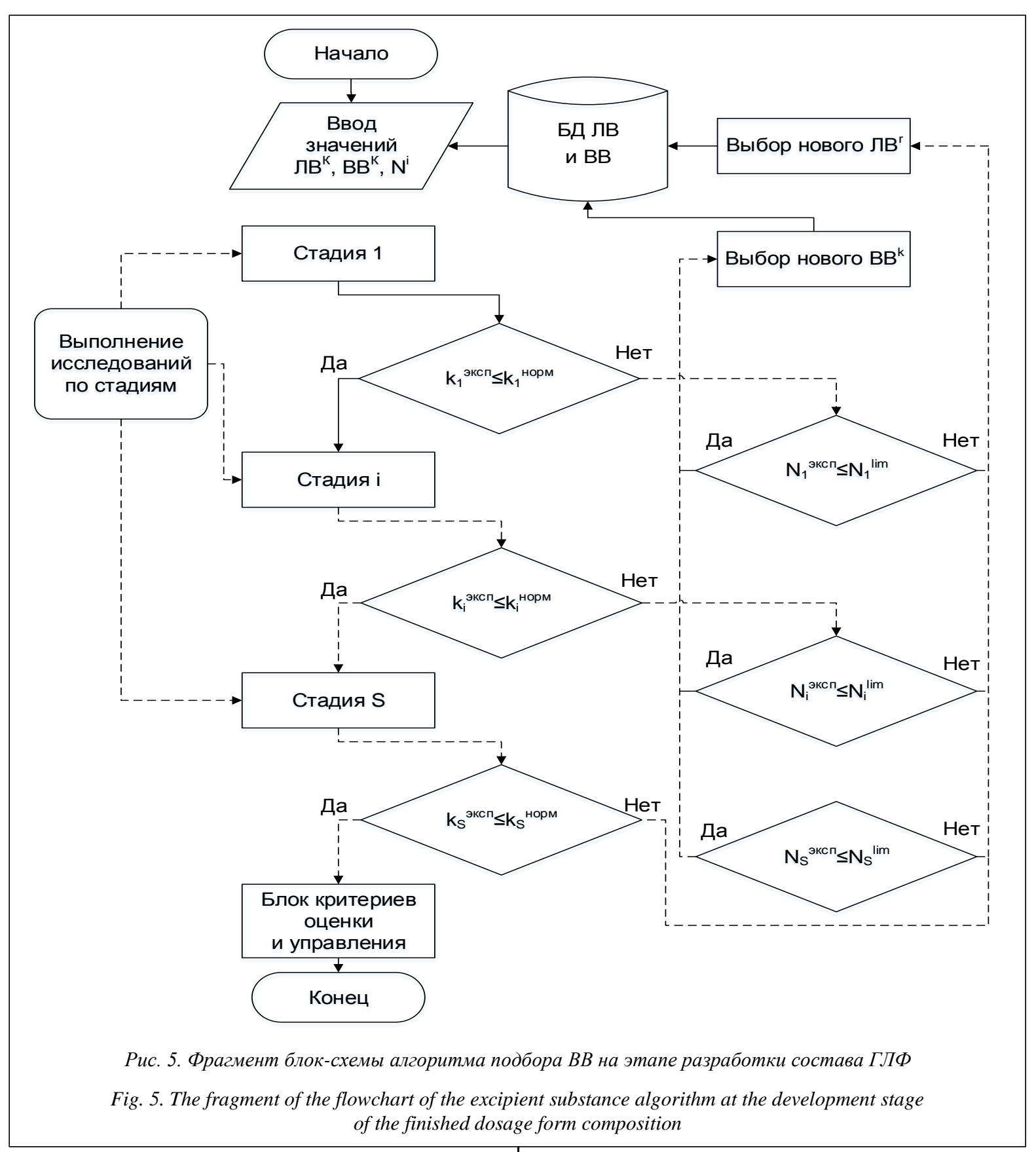

Было указано, что одной из важнейших задач, решаемых на этапе разработки состава ГЛФ, является подбор ВВ. Для этого используется БД ВВ, входящая в структуру интеллектуальной системы управления. В процессе подбора ВВ исследователю необходимо производить поиск активных фармацевтических субстанций и ВВ по ряду критериев, таких как способ применения, лекарственная форма, подробная информация о веществе и поставщиках и т.д.

На основе фактических данных были выделены основные сущности и связи между ними и построена модель «сущность-связь», для которой в качестве основных сущностей были выделены следующие: ВВ, фармацевтическая субстанция, лекар- ственная форма, способ применения, класс ВВ, поставщик, синоним, показатель контроля.

Связь сущностей между собой показана на рисунке 6.

Данная модель наглядно отражает связь между отдельными сущностями, а на ее базе построена модель реляционной БД ВВ.

Для построения схемы данных (см. http://www. swsys.ru/uploaded/image/2018-4/2018-4-dop/17.jpg) проводится их нормализация, так как в реляционной БД недопустимо использование связей «многие-ко-многим». Такие связи заменяются промежуточными таблицами, а связь таблиц с сущностями и промежуточных осуществляется с помощью связи «один-ко-многим». Также для связи таблиц в 


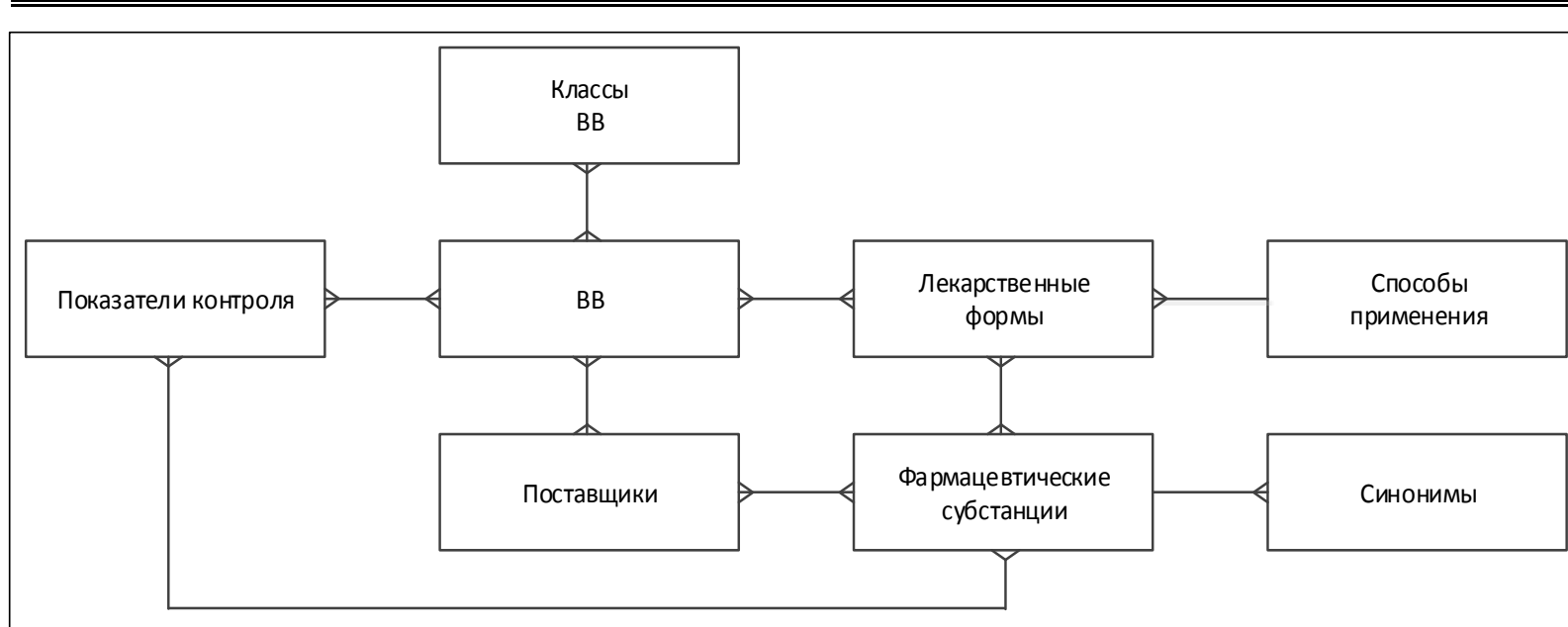

Рис. 6. Модель «сущиность-связь»

Fig. 6. The "Entity-Relationship" model

БД выделены ключевые поля в виде первичных и внешних ключей. Это необходимо для обеспечения целостности данных в системе, а также для ускорения поиска зависимостей внутри БД.

Для обеспечения доступа к БД из внешних систем необходимо было разработать АРІ. Стоит отметить, что при разработке web-приложения с использованием технологий SPA (single page application) можно использовать одинаковый API и для интерфейса приложения, и для внешних систем. Такой подход заметно сокращает временные затраты, необходимые для разработки и дальнейшей поддержки системы, так как отсутствует необходимость поддерживать два интерфейса одновременно. Однако при таком подходе необходимо организовать механизм авторизации для внешних систем. На данный момент наиболее распространены два механизма: OAuth и JWT (JSON WebTokens). Несмотря на то, что второй механизм несколько проще в реализации, он имеет ряд недостатков, связанных, во-первых, с увеличением количества данных, отправляемых сервером с каждым запросом, а во-вторых, с проблемами инвалидации ранее выданных ключей доступа. Поэтому, несмотря на более сложный процесс интеграции механизма OAuth, на текущий момент он выглядит более надежным решением, по крайней мере, в рамках данной задачи.

При разработке SPA-приложения необходимо реализовать две раздельные части системы: клиентскую, с которой будет взаимодействовать конечный пользователь, и серверную, представляющую в случае разработки БД ВВ сервер приложения, который получает данные из конечной СУБД и передает их клиенту.

Для разработки web-приложений можно использовать большое количество высокоуровневых фреймворков. Наиболее распространенными из них на данный момент являются Angular, React и Vue [11].
Vue - это программная платформа с открытым исходным кодом, написанная на языке JavaScript, которая позволяет создавать пользовательские интерфейсы с использованием парадигмы реактивного программирования. Приложение, разработанное с использованием vue, может быть интегрировано в уже функционирующее веб-приложение и использоваться отдельно.

При разработке vue-приложений используется компонентный подход. Каждый элемент интерфейса, будь то кнопка или целая страница, в браузере может быть представлен в качестве компонента. В свою очередь, каждый компонент может использовать внутри себя другие компоненты и т.д.

При разработке интерфейса были определены следующие требования:

- возможность просмотра, редактирования и поиска информации о ВВ, фармацевтических субстанциях и поставщиках;

- возможность поиска ВВ по заданным критериям;

- возможность интеграции с внешними системами для актуализации и добавления новых данных из внешних источников;

- возможность использования системы как локально, так и в мультикомпьютерном режиме;

- необходимость системы авторизации и разграничения прав пользователей.

На основании данных требований можно определить основные разделы интерфейса разрабатываемого приложения:

- поиск;

- работа с веществами: с ВВ и с фармацевтическими субстанциями;

- управление поставщиками;

- управление пользователями.

Как было сказано ранее, использование ORM позволяет работать с БД из разрабатываемого приложения без оглядки на конкретную СУБД. Это 
важное свойство системы в момент ее интеграции в уже существующую инфраструктуру.

Следующим шагом является применение так называемых встраиваемых СУБД, например SQLite.

Использование SQLite позволяет минимизировать расход памяти и процессорного времени приложением, так как БД будет запущена только в момент работы приложения с ней.

Представим фрагмент кода для создания таблицы ВB:

1. Schema::create('substances', function (Blueprint \$table) \{

2. $\quad$ \$table->increments('id');

3. \$table->string('name');

4. \$table->enum('origin', ['synthetic', 'organic']);

5. \$table- $>$ string('article no', 100);

6. \$table->text('description')->nullable();

7. \$table->timestamps();

8. \$table->softDeletes();

9. \});

Таким образом, указанный выше код генерирует следующий SQL-запрос для создания таблицы:

1. CREATE TABLE `substances` (

2. 'id' int(10) un-

signed NOT NULL AUTO_INCREMENT,

3. 'name' var-

char(255) COLLATE utf8mb4 unicode ci NOT NULL,

4. 'origin 'enum('synthetic','organ-

ic') COLLATE utf8mb4 unicode ci NOT NULL,

5. 'article no' var-

char(100) COLLATE utf8mb4_unicode_ci NOT NULL,

6. 'description' text COLLATE utf8mb4 unicode_ci,

7. 'created_at' timestamp NULL DEFAULT NULL,

8. 'updated at' timestamp NULL DEFAULT NULL,

9. 'deleted_at' timestamp NULL DEFAULT NULL,

10. PRIMARY KEY ('id')

11. ) ENGINE=InnoDB AUTO INCREMENT=49 DEFAU

LT CHARSET $=$ utf8mb4 COLLATE $=$ utf8mb4_unicode_ci;

Немаловажным при проектировании БД является работа с внешними ключами и индексами. Eloquent ORM предоставляет все необходимые для этого инструменты. Например, таблицу связи поставщика и ВВ можно создать следующим обра3ом:

1. Schema::create('substance_supplier', function(Blueprint \$table) \{

2. \$table-> unsignedInteger('substance_id')

3. $\quad->$ references('id')

4. $\quad->$ on('substances')

5. $\quad \rightarrow$ onDelete('cascade');

\$table-> unsignedInteger('supplier_id')

$->$ references('id')

$->$ on('suppliers')

$->$ onDelete('cascade');

10. \$table->unique(['substance id', 'supplier_id']); 11. \});

В заключение отметим, что в данной работе рассмотрено решение ряда задач применения системного анализа для информационной поддержки этапа разработки состава твердых лекарственных форм.
Построена информационная модель в номинации IDEF0 жизненного цикла разработки состава ГЛФ, начиная от поиска и синтеза активной фармацевтической субстанции.

На основе системного подхода рассмотрены основные проблемы информационной поддержки реализации принципа QbD на этапе разработки состава ГЛФ.

Разработаны системные теоретико-множественные, информационные и математические модели для теоретического обоснования информационной поддержки этапа разработки состава лекарственной формы.

Показано применение критериального подхода для формирования глобального критерия и локальных критериев при подготовке управленческих решений при разработке состава ГЛФ на основе системы химического, микробиологического и технологического контроля. Кроме того, детально рассмотрены информационные и программные средства построения базы ВВ, играющие определенную роль в разработке и формировании технологической платформы.

\section{Лumepamypa}

1. Решетников В.Н., Мамросенко К.А. Информационные технологии в здравоохранении: развитие региональных систем // Программные продукты, системы и алгоритмы. 2016. № 1. URL: http://swsys-web.ru/information-technology-in-health-caredevelopment-of-regional-systems.html (дата обращения: 03.10.2018).

2. Энде Д.Дж. Производство лекарственных средств. Химическая технология от R\&D до производства; [пер. с англ.]. СПб: Профессия, 2015. 1280 с

3. Watson T.J.N., Nosal R., am Ende D., Bronk K., Mustakis J., O’Connor G., Charles L. Santa Maria Ch.L. API quality by design example from the torcetrapib manufacturing process. J. of Pharmaceutical Innovation, 2007, vol. 2, no. 3-4, pp. 71-76. DOI: 10.1007/s12247-007-9015-x.

4. Таптунов В.Н., Екимов С.Д., Гусева Е.В. Информационная интеллектуальная система для разработки схем производства твердых лекарственных форм // Программные продукты и системы. 2008. № 4. С. 160-161.

5. Волкова В.Н., Денисов А.А. Теория систем и системный анализ. М., 2012. 679 с.

6. Rubin E., Tummala S., Both D., Wang C. and Delany E. Emerging technologics supporting chemical process R\&D and their increasing impact on productivity in the pharmaceuticalindustry, Chem. Rev., 2006, vol. 7, no. 106, pp. 2794-2810. DOI: 10.1021/cr040674i.

7. Levin M., ed. Pharmaceutical Process Scale-Up. Drugs and the Pharm. Sc. Ser., NY, Marcel Dekker Publ., 2006, vol. 157, 538 p.

8. Корнюшко В.Ф., Панов А.В., Богунова И.В., Николаева О.М., Флид А.А. Применение системного подхода для построения информационного пространства разработки состава готовых лекарственных форм // Прикладная информатика. 2018. T. 13. № 3. C. $83-100$.

9. Костров А.В., Головина Е.А., Корнюшко В.Ф., Кузин Р.Е. Подход к совершенствованию средств управления развитием персонала в автоматизированной системе предприятия

// Информационные и телекоммуникационные технологии. 2015. № 28. С. $35-41$.

10. Мешалкин В.П. Экспертные системы в химической технологии. М.: Химия, 1995. 367 с.

11. Алькаев Р.Р., Учватов С.А., Толстых Н.Д. Обзор возможностей EMS SQL managerfor SQL server // Apriori. Cep.: Естественные и технические науки. 2016. № 3. С. 14. 


\section{Information support of the development stage of solid dosage form composition}

V.F. Kornyushko ${ }^{1}$, Dr.Sc. (Engineering), Professor, vfk256@mail.ru

O.M. Nikolaeva ${ }^{1}$, Assistant, polyakova@mitht.ru

I.V.Bogunova ${ }^{1}$, Researcher, irina_bogunova@mail.ru

A.A. Flid ${ }^{1}$, Leading Programmer, a.a.flid@gmail.com

A.A. Grebenshchikov ${ }^{1}$, Master of Science, me@ package.su

${ }^{1}$ Russian Technological University (M.V. Lomonosov Institute of Fine Chemical Technologies),

Moscow, 119471, Russian Federation

Abstract. The paper discusses the use of the system analysis for the information support of the development stage of the solid dosage form composition. It also presents the informational description diagram of the development and production life cycle of the medications in the IDEF0 nomination. The information support system for the composition development phase in the life cycle is based on a new QbD approach (Quality by Design), which should be implemented based on a medicine target profile - TPP (Target Product Profile).

Technological parameters characterizing the main properties of the obtained intermediate products, CPP (Critical Process Parameter), were chosen as criteria for evaluating the optimization quality at all development stages.

The paper describes the scheme of the intellectual control system for the development stage of the finished dosage form composition.

The authors propose two algorithms for research management: one is for selecting support substances, and the second is for selecting optimal technological parameters for each stage.

The paper considers in detail the construction of the parameter-fitting algorithm of all processes involved in obtaining the optimal composition of the finished medicines based on the multi-click regression model. It also considers the construction of the support substances database that is included in the intelligent control system structure. There are diagrams showing the basic sets of entities and the relationships between them. Among other things, there are some program code fragments that support the theoretical substantiation of the conducted research.

Keywords: system analysis, formulation development, information support, IDEF0 nomination, QbD approach, TPP profile, criterion, information model, database, management system.

\section{References}

1. Reshetnikov V.N., Mamrosenko K.A. Information technologies in healthcare: the development of regional systems. Software Products, Systems and Algorithms. 2016, no. 1. Available at: http://swsys-web.ru/information-technology-in-healthcare-development-of-regional-systems.html (accessed October 3, 2018).

2. Ende D.J. Chemical Engineering in the Pharmaceutical Industry: R\&D to Manufacturing. Wiley Publ., 2010, 904 p. (Russ. ed.: St. Petersburg, Professiya Publ., 2015, 1280 p.).

3. Watson T.J.N., Nosal R., am Ende D., Bronk K., Mustakis J., O’Connor G., Charles L. Santa Maria Ch.L. API quality by design example from the torcetrapib manufacturing process. J. of Pharmaceutical Innovation. 2007, vol. 2, no. 3-4, pp. 71-76. DOI: 10.1007/s12247-007-9015-x.

4. Taptunov V.N., Ekimov S.D., Guseva E.V. An information intellectual system for the development of schemes for the production of solid medicinal forms. Software \& Systems. 2008, no. 4, pp. 160-161 (in Russ.).

5. Volkova V.N., Denisov A.A. Theory of Systems and System Analysis. Moscow, 2012, 679 p.

6. Rubin E., Tummala S., Both D., Wang C., Delany E. Emerging technologies supporting chemical process R\&D and their increasing impact on productivity in the pharmaceuticalindustry. Chem. Rev. 2006, no. 106, pp. 2794-2810. DOI: 10.1021/cr040674i.

7. Levin M. Pharmaceutical Process Scale-Up. 2nd ed. Marcel Dekker Publ., 2006, vol. 157, 538 p.

8. Kornyushko V.F., Panov A.V., Bogunova I.V., Nikolaeva O.M., Flid A.A. System approach to informational support of pharmaceutical development of finished medicinal products. Applied Informatics. 2018, vol. 13, no. 3, pp. 83-100 (in Russ.).

9. Kostrov A.V., Golovina E.A., Kornyushko V.F., Kuzin R.E. Approach to improvement of personnel development management tools in the automated system of the enterprise. Information and Telecommunication Technologies. 2015, no. 28, pp. 35-41 (in Russ.).

10. Meshalkin V.P. Expert Systems in Chemical Technology. Moscow, Khimiya Publ., 1995, 367 p.

11. Alkaev R.R., Uchvatov S.A., Tolstykh N.D. Overview of EMS SQL manager for SQL server. Apriori. Ser.: Natural and Technical Sciences. 2016, no. 3, p. 14. 